This department welcomes short notes and problems believed to be new. Contributors should include solutions where known, or background material in case the problem is unsolved. Send all communications concerning this department to I. G. Connell, Department of Mathematics, McGill University, Montreal, P. C.

\title{
REMARK ON CO-NULL MATRICES
}

\section{M.S. Macphail ${ }^{1}$}

A well-known theorem of Copping [2] states that a conservative matrix with a bounded left inverse cannot evaluate a bounded divergent sequence. (Definitions are given in the next paragraph.) A proof was given by Parameswaran [3, Theorem 6. 1], using only the simplest Banach-space ideas. This proof, however, is valid only for co-regular methods; it was stated in [3, Theorem 6.2] that a co-null matrix cannot have a bounded left inverse, but the proof there given is incorrect, as it uses for co-null methods a theorem established only for co-regular. It would be desirable to have a short independent proof of this known result, which excludes co-null matrices from consideration in Copping's theorem. This is furnished by the slightly more general result given below.

The matrix $A=\left(a_{n k}\right)$ is conservative if the column limits and the row-sums limit exist, and the row-norms are bounded. We denote the column limits by $a_{k}=\lim _{n} a_{n k}$, the row sums by $\alpha_{n}=\Sigma_{k} a_{n k}$, the row-sum limit by $\alpha_{0}=\operatorname{Iim} \alpha_{n}$, and the row norms by $\rho_{n}=\Sigma_{k}\left|a_{n k}\right|$. Any matrix with $\rho_{n}$ bounded is called a bounded matrix. We denote the Banach space of convergent

1 Written while the author was a member of the Summer Research Institute, Kingston, 1964. 
sequences by $c$, of bounded sequences by $m$, with the usual norms. If the matrix $A$ is conservative, it maps $c$ into $c$, if bounded, $m$ into $m$, continuously in each case. We define $x(A)=a_{0}-\Sigma a_{k}$; then provided the matrix $A$ is conservative we call it co-null if $\chi(A)=0$, co-regular otherwise.

IHEOREM. Let $A$ be co-null and $B$ bounded. Then if the product $\mathrm{C}=\mathrm{BA}$ is conservative, it must be co-null.

Remark 1. If under our hypothesis we have $B A=I$, there would be an immediate contradiction, thus proving the statement in the opening paragraph, that a co-null matrix cannot have a bounded left inverse.

Remark 2. If $B$ were conservative, the result would follow immediately by the formula $\chi(B A)=\chi(B) \times(A)[4$, p. 398].

Proof of theorem. Denote the columns of $A$ by $A_{1}, A_{2}, \ldots$, and the (column) vector $\left\{\alpha_{n}\right\}$ by $\alpha$. Let $S_{k}=A_{1}+\ldots+A_{k}$; then $A$ is co-null if and only if $S_{k}$ converges weakly to $\alpha$ in $c[1$, p. 136-137]. We assume this. Now the left multiplier $B$ may be regarded as a continuous operator from $c$ into $m$, mapping the columns of $A$ into the columns of C. By a theorem of Banach [1, p. 143] the images $\mathrm{BS}_{\mathrm{k}}$ converge weakly to $\mathrm{B} \alpha$ in $\mathrm{m}$. Since the terms of a sequence are linear functionals, we have that $\mathrm{BS}_{\mathrm{k}}$ converges termwise to $B \alpha$, and so $B \alpha$ is the vector composed of the row sums of $C$. As we are assuming $C$ conservative, we have each $\mathrm{BS}_{\mathrm{k}} \in \mathrm{c}$, and $\mathrm{B} \alpha \in \mathrm{c}$. Then, since every linear functional on $c$ is a restriction of a linear functional on $\mathrm{m}$, we have in particular that $\lim \mathrm{BS}_{\mathrm{k}}$ approaches $\lim \mathrm{B} \alpha$, and so $C$ is co-null, as was to be proved.

There is no corresponding theorem for $A B$, as shown by the example [4] 


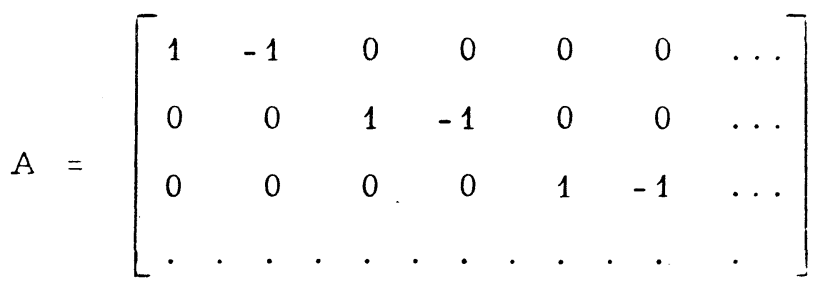

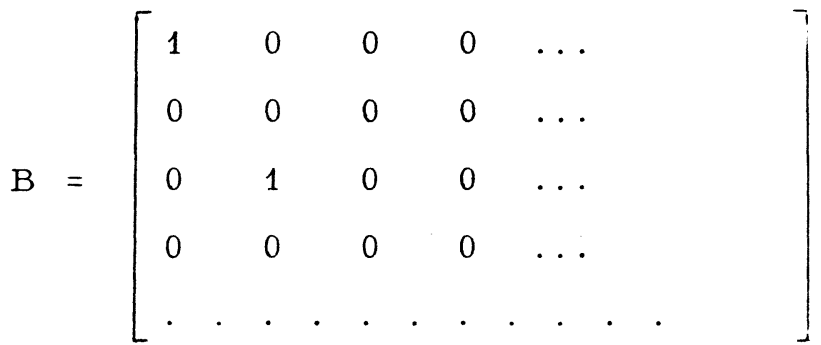

where $A B=I$.

\section{REFERENCES}

1. S. Banach, Théorie des opérations linéaires.

2. J. Copping, $K$ matrices which sum no bounded divergent sequence, Journal of the London Mathematical Society, 30 (1955), 123-127.

3. M.R. Parameswaran, Some applications of Banach functional methods to summability, Proceedings of the Indian Academic Society, (A) 45 (1957), 377-384.

4. A. Wilansky and $\mathrm{K}$. Zeller, The inverse matrix in summability: reversible matrices, Journal of the London Mathematical Society, 32 (1957), 397-408.

Carleton University,

Ottawa, Canada 